\title{
Investigation of Porous Zn Growth Mechanism during Zn Reactive Sputter Deposition
}

\author{
M.A. Borysiewicz ${ }^{a, *}$, T. WojCIEChOWski ${ }^{b}$, E. DyNOWskA $^{a, b}$, E. KAMiŃsKA $^{a}$ \\ AND A. PIOTROWSKA ${ }^{a}$ \\ ${ }^{a}$ Institute of Electron Technology, al. Lotników 32/46, 02-668 Warsaw, Poland \\ ${ }^{b}$ Institute of Physics, PAS, al. Lotników 32/46, 02-668 Warsaw, Poland
}

(Received March 22, 2013)

\begin{abstract}
Ar-O-Zn plasma discharges created during DC reactive magnetron sputtering of a $\mathrm{Zn}$ target and $\mathrm{RF}$ reactive magnetron sputtering of a ceramic $\mathrm{ZnO}$ target were investigated and compared by means of the Langmuir probe measurements in order to determine the mechanism of growth of porous $\mathrm{Zn}$ films during DC-mode Zn reactive sputtering. The power supplied to the magnetrons during the sputtering was kept at $125 \mathrm{~W}$ and the plasma was characterised as a function of oxygen content in the sputtering gas mixture, ranging from 0 to $60 \%$ for two gas pressures related to porous $\mathrm{Zn}$ film deposition, namely $3 \mathrm{mTorr}$ and $5 \mathrm{mTorr}$. Based on the correlation of plasma properties measurements with scanning electron microscope imaging and X-ray diffraction of the films deposited under selected conditions it was found that the growth of porous, polycrystalline Zn films was governed by high electron density in the plasma combined with a high electron temperature and an increased energy of the ions impinging on the substrate.
\end{abstract}

DOI: $10.12693 /$ APhysPolA.125.1144

PACS: 52.70.-m, 81.15.Cd, 77.55.hf, 81.10.Pq, 52.27.Cm

\section{Introduction}

There is a growing interest in the fabrication of high surface-to-volume ratio materials due to the strong development of absorption-based devices such as dye sensitized solar cells, as well as chemical and biological sensors. Metal oxides, in particular $\mathrm{ZnO}$ have been selected as materials of choice for such applications due to their exceptionally chemically active surfaces enabling their easy functionalization. Furthermore, a wide array of morphologies has been developed including nanoparticles [1], nanowires [2], nanobelts [3] and more exotic shapes as nanoforests [4]. $\mathrm{ZnO}$ nanostructures with porous morphology were recently demonstrated by our group using reactive magnetron $\mathrm{Zn}$ sputtering [5]. Therein it was shown that under specific process conditions applied to reactive DC-sputtering of a $\mathrm{Zn}$ target (process pressures $\leq 3$ and $17 \%$ oxygen content in the sputtering mixture) a porous $\mathrm{Zn}$ film grew instead of the expected dense $\mathrm{ZnO}$ film. Subsequent RTP annealing in an oxygen flow at $400{ }^{\circ} \mathrm{C}$ transformed the $\mathrm{Zn}$ film into porous $\mathrm{ZnO}$. It has to be noted that to the best of our knowledge these nanostructures are the only sputter deposited $\mathrm{ZnO}$ nanostructures, profiting from the high level of purity and process control of this technique as well as from its scalability and compatibility with industrially used coating processes. Therefore, the understanding of their growth mechanism is relevant as it may be applied to the sputtering of other material systems leading to the creation of new nanostructures.

*corresponding author; e-mail: mbory@ite.waw.pl
In this paper we discuss the mechanism standing behind the growth of porous $\mathrm{Zn}$ during $\mathrm{Zn}$ reactive sputtering, using observations of the electrical properties of the plasma, studied by means of a Langmuir probe and optical emission spectroscopy, respectively. A wide range of growth conditions is applied in order to analyse which yield porous $\mathrm{Zn}$ films and which give dense $\mathrm{ZnO}$ films. As a reference, $\mathrm{RF}$ sputtering of a $\mathrm{ZnO}$ target was chosen as it was expected that, in contrast to DC-mode Zn sputtering, there would be minute changes in its sputtering behaviour and the structure of the deposited films under the whole range of process parameters used in the experiment. The films were grown on $\mathrm{Si}(111)$ substrates and subjected to structural characterisation using X-ray diffraction (XRD) and cross-sectional scanning electron microscopy (SEM).

\section{Experimental details}

An $\mathrm{Ar}-\mathrm{O}-\mathrm{Zn}$ plasma discharge was maintained through sputtering from $4 \mathrm{~N}$-pure $\mathrm{Zn}$ and $\mathrm{ZnO}$ targets in a $6 \mathrm{~N}$-pure argon-oxygen mixture from an unbalanced magnetron in DC and RF modes, respectively. The target-to-substrate distance was $15 \mathrm{~cm}$ and the substrate was positioned under the target, with the target face at $60^{\circ}$ angle to the substrate surface. The base pressure in the chamber prior to deposition was of the order of $10^{-7}$ Torr. The power at the target was kept constant at $125 \mathrm{~W}$, both $\mathrm{DC}$ and $\mathrm{RF}$ (with $0 \mathrm{~W}$ reflected power in the latter case). The amount of oxygen in the sputtering gas mixture was changed by varying the oxygen flow from 0 to $15 \mathrm{sccm}$ while maintaining a constant Ar flow of $10 \mathrm{sccm}$, which resulted in changes in oxygen content in the mixture from 0 to $60 \%$. 


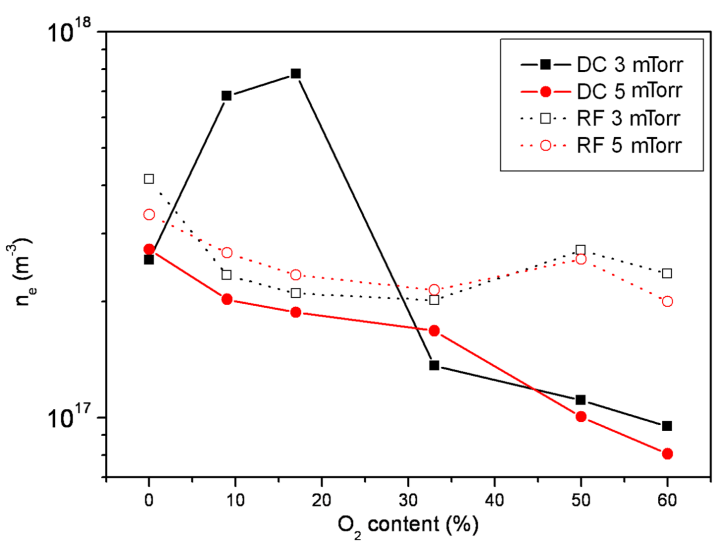

Fig. 1. The changes in $n_{\mathrm{e}}$ with the addition of oxygen into the gas mixture observed for DC and RF plasmas at $3 \mathrm{~m}$ Torr and 5 mTorr.

The samples fabricated in the experiment.

TABLE

\begin{tabular}{c|c|c|c|c}
\hline $\begin{array}{c}\text { Sample } \\
\text { No. }\end{array}$ & $\begin{array}{c}p \\
{[\mathrm{~m} \text { Torr }]}\end{array}$ & $\begin{array}{c}\mathrm{O}_{2} \text { content } \\
{[\%]}\end{array}$ & Mode & $\begin{array}{c}\text { Growth rate } \\
{[\AA / \mathrm{s}]^{*}}\end{array}$ \\
\hline 1 & 3 & 17 & $\mathrm{DC}$ & 4.8 \\
2 & 5 & 17 & $\mathrm{RF}$ & 1.1 \\
3 & 5 & 60 & $\mathrm{RF}$ & 0.9 \\
4 & 5 & 17 & $\mathrm{DC}$ & 2.2 \\
5 & 5 & 60 & $\mathrm{DC}$ & 1.7 \\
\hline
\end{tabular}

${ }^{*}$ Film thickness derived from SEM measurements.

The sputtering was performed at two constant total gas pressures: 3 and 5 mTorr. From previous experiments it was known that the highest pressure for porous film growth in the system used was 3 mTorr, therefore this and 5 mTorr pressures were chosen in order to enable comparison between conditions enabling porous film growth and conditions under which the film grows as a dense one. $I-V$ characteristics of the plasma were taken using a Hiden Analytical ESPion Langmuir Probe positioned close to the substrate. The probe tip was made of tungsten and the voltage applied to the probe was changed in the range of $(-200)-(+100) \mathrm{V}$ with a step of $0.2 \mathrm{~V}$. Before each measurement an electron pre-cleaning step was applied by biasing to $+20 \mathrm{~V}$ for $120 \mathrm{~ms}$. The ESPion Langmuir probe is equipped in a passive high-frequency compensation electrode with a string of inductors in order to remove the $\mathrm{AC}$ component between the plasma and the probe tip appearing in $\mathrm{RF}$ plasmas. Based on the results of plasma density measurements performed using the Langmuir probe (see Fig. 1), five process parameter sets were chosen fully spanning the conditions' space, under which thin films were deposited to study the relationships between the plasma parameters and the resulting film characteristics. One parameter set was chosen to yield porous $\mathrm{Zn}$ thin film growth (3 mTorr at low oxygen concentration in DC mode as found earlier [5]) and four others were chosen for low and high oxygen contents both in DC and RF at 5 mTorr.
Table summarizes the deposition parameters of the films. The films were deposited onto $1 \mathrm{~cm} \times 1 \mathrm{~cm} \mathrm{Si}$ (111) substrates kept at floating potential. Initially, the silicon was degreased by boiling in hot trichloroethylene, acetone and IPA, rinsed with deionised water and dried with nitrogen gas. Prior to deposition the substrates were deoxidised by immersing for 30 seconds in a buffered HF bath, followed by rinsing and drying as previously. The substrates were not intentionally heated during the deposition process. The film structure was subsequently investigated using a Phillips X'Pert MPD Pro diffractometer working in the $\theta-2 \theta$ geometry and a Zeiss Auriga scanning electron microscope in cross-section views.

\section{Results}

\subsection{Langmuir probe measurements}

In a low-density plasma, such as the one created during sputtering, the approximation of electric quasi-neutrality generally holds. In this case, the electron density, $n_{\mathrm{e}}$, obtained using the Langmuir probe via the determination of electron temperature, plasma potential, and electron saturation current, may be also thought of as equal to the density of positive ions in the plasma, $n_{i+}$. This is the case we assume here and will use the determined electron density as referring both to the negatively charged electrons and positively charged ions and we will therefore call it simply "plasma density". In the reported experiment, the density has similar characteristics in the function of oxygen content for different pressures for the RF mode, nevertheless they differ significantly in the DC mode (see Fig. 1).

In $\mathrm{RF}$ mode the density values are almost constant, being in the range of $(2-4) \times 10^{17} / \mathrm{m}^{3}$ with only small changes in value with the addition of oxygen and very minute changes with the rise of total pressure. The situation is considerably different in DC mode sputtering, where the changes are very pronounced. The density for pure $\mathrm{Zn}$ sputtering regardless of the total pressure lies around $2.5 \times 10^{17} / \mathrm{m}^{3}$ and with the addition of small amounts of oxygen (to $9 \%$ and $17 \%$ in the mixture) it rises to around $6.8-7.8 \times 10^{17} / \mathrm{m}^{3}$, respectively, in the case of $3 \mathrm{mTorr}$ pressure, which are the conditions present during the growth of the porous $\mathrm{Zn}$ films. In the case of 5 mTorr pressure, the changes are smaller and different as the density falls slightly with the addition of oxygen. This effect may be explained based on an assumption, that at small amounts and low pressures ( $\leq 3$ mTorr) the oxygen does not poison the surface of the target, but stays in the plasma, taking part in the sputtering process, leading also to an increase in the sputtering rate of the material.

From Table it can be seen that the deposition rate can be connected to the plasma density in the DC mode, which strengthens the validity of this assumption.

Furthermore, as it is shown by XRD studies presented in Sect. 3.3, the film grown under such conditions is a 
film dominated by $\mathrm{Zn}$, not $\mathrm{ZnO}$, which leads to the conclusion that in this case limited reaction between $\mathrm{O}$ and $\mathrm{Zn}$ takes place, which would agree with the hypothesis of O-assisted sputtering.

With further addition of oxygen, above $33 \%$ content, the electron concentrations drop to around $1.5 \times 10^{17} / \mathrm{m}^{3}$ at first and for $60 \%$ content they reach 0.9 and $0.8 \times 10^{17} / \mathrm{m}^{3}$ in the case of $3 \mathrm{mTorr}$ and $5 \mathrm{mTorr}$, respectively. These values are lower than in the case of RF sputtering. This effect has to be due to an increase in the poisoning rate of the target surface with the addition of oxygen, with a faster poisoning for larger absolute number of oxygen atoms, i.e. at 5 mTorr. This increase in poisoning rate observable at $33 \%$ can be correlated to the drop in cathode voltages observable for $33 \%$ oxygen content at both pressures, an effect which does not take place in RF mode sputtering (see Fig. 2). It has to be noted that in the system used, $60 \%$ at 5 mTorr pressure is the oxygen content above which stable $\mathrm{Zn}$ sputtering in DC mode becomes impossible due to overwhelming target poisoning.

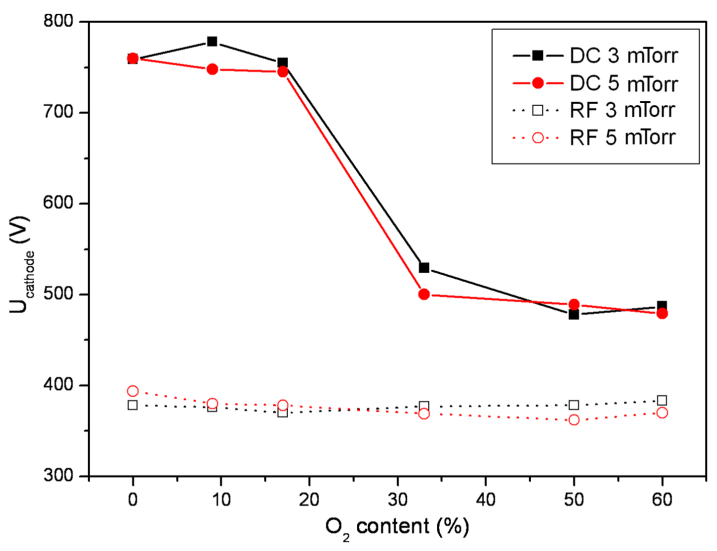

Fig. 2. The changes in cathode voltage with the addition of oxygen into the gas mixture observed for DC and RF plasmas at 3 mTorr and 5 mTorr.

The electron temperature, $T_{\mathrm{e}}$, in the case of DC sputtering lies at the level of around $1 \mathrm{eV}$ (see Fig. 3) for pure $\mathrm{Zn}$ sputtering and for sputtering at oxygen contents equal to and above $33 \%$. However, at $9 \%$ and $17 \%$ significant changes occur in the characteristic. A rise (drop) in the temperature is observed to $2.7 \mathrm{eV}(0.4 \mathrm{eV})$ for 3 mTorr ( 5 mTorr). The $T_{\mathrm{e}}$ in the case of RF sputtering at 3 mTorr has a similar characteristic with a peak observed at $17 \%$ and at $5 \mathrm{~m}$ Torr the characteristic is completely different, with the highest $T_{\mathrm{e}}=4.2 \mathrm{eV}$ observable for sputtering in pure argon.

The plasma generated at the parameters resulting in porous $\mathrm{Zn}$ deposition, i.e. $17 \%$ oxygen and $3 \mathrm{mTorr}$ is the highest energetic of the DC plasmas observed in the experiment, not only due to the highest electron temperature, but also due to the largest energy of ions impinging onto the substrate observed for DC mode (see Fig. 4). This energy, extracted from the difference between the

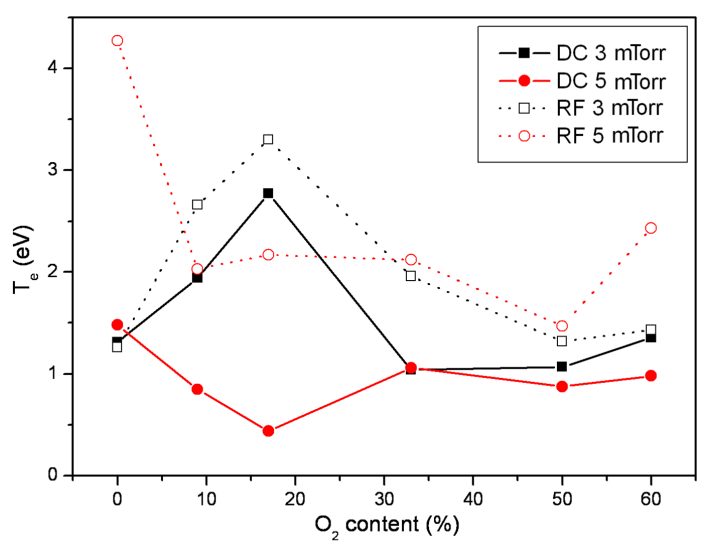

Fig. 3. The changes in $T_{\mathrm{e}}$ with the addition of oxygen into the gas mixture observed for DC and RF plasmas at $3 \mathrm{~m}$ Torr and 5 mTorr.

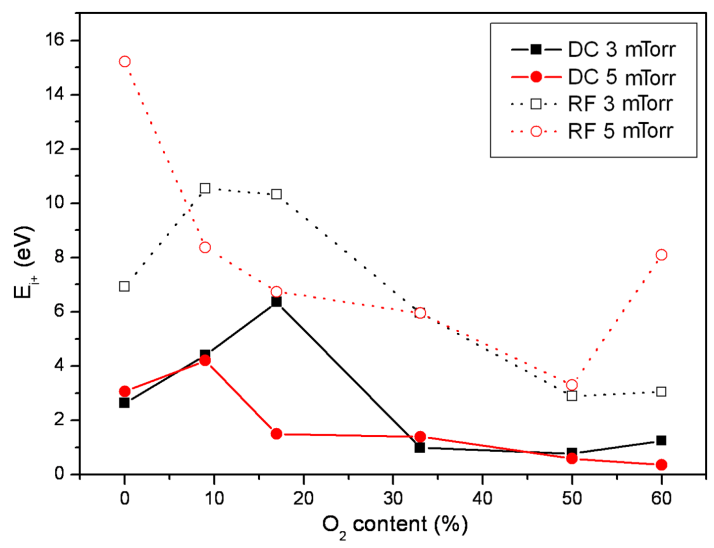

Fig. 4. The changes in $E_{i+}$ with the addition of oxygen into the gas mixture observed for DC and RF plasmas at $3 \mathrm{mTorr}$ and $5 \mathrm{~m}$ Torr.

plasma and float potentials measured is higher in the RF mode. This is so, even though the substrate self-bias in RF mode (i.e. the float potential) is an order of magnitude higher than in the case of the DC mode (roughly $20 \mathrm{~V}$ vs. $2 \mathrm{~V}$, respectively).

\subsection{Scanning electron microscopy}

Figure 5 presents scanning electron microscopy cross-section images for all samples in the experiment, as presented in Table. It can be seen that the morphology of samples is quite varied, in particular sample 1 exhibits the expected porous microstructure, and samples $2,3,4$ and 5 exhibit different columnar microstructures. Comparing the plasma properties of samples 1 and 2 grown at similar process parameters, with the only difference lying in the pressure, it can be seen that $T_{\mathrm{e}}, E_{i+}$, and $n_{\mathrm{e}}$ are higher for sample 1 . This may lead to a conclusion that the growth of porous films is promoted by high plasma density and temperature possibly yielding increased collisions of the species exiting the target as well as by high enough ion energy to impinge the species on the substrate 


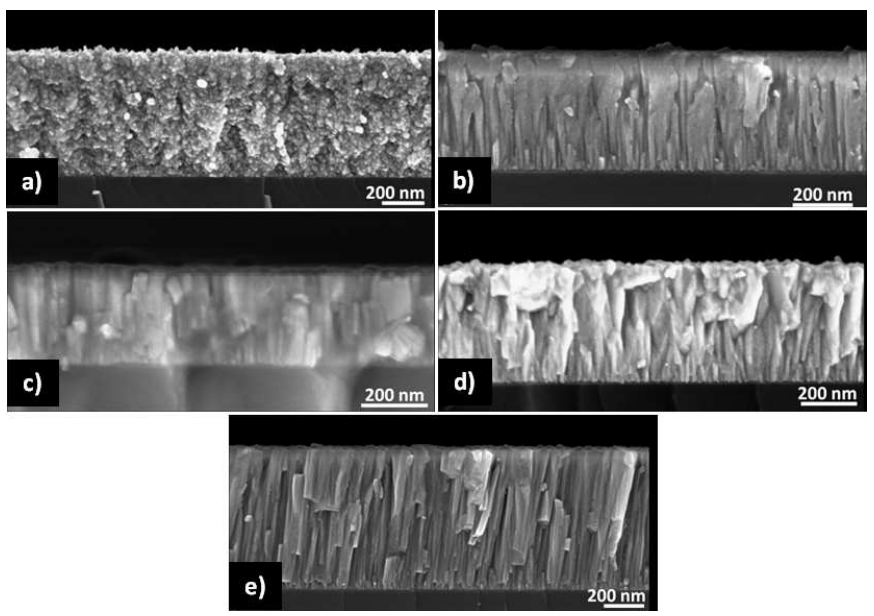

Fig. 5. Scanning electron microscope cross-section images of the samples produced in the experiment, a) shows sample $1, \mathrm{~b})-2, \mathrm{c})-3, \mathrm{~d})-4$ and e) -5 . The Si substrate is at the bottom of the image. The values of $n_{\mathrm{e}}$, given in the units of $10^{17} \mathrm{~m}^{-3}$, measured for respective samples during deposition were the following: $1-7.77,2-2.34,3-2.00,4-1.87$, and $5-0.81$.

in the point of contact, deteriorating surface adatom mobility.

Samples 2 and 3 have been grown at conditions with similar plasma parameters, however with sample 3 being grown at higher oxygen content. The microstructure is very similar, however sample 3 shows columns of a constant width, while in sample 3 the columns get wider when closer to the substrate. Sample 4 shows features similar as those visible in samples 2 or 3 . On the other hand, sample 5 while still maintaining the columnar microstructure, has the column growth axis significantly tilted with respect to the substrate surface, at $75^{\circ}$. As the magnetron is tilted at $60^{\circ}$ and the samples were positioned close to the magnetron and not on the axis of the magnetron, this angle may be equal to the angle of sight of the magnetron for the sample. The only plasma parameter significantly different for this sample from the ones for other samples is the plasma density, being the lowest of all. This may yield longer mean free paths of the sputtered species, leading to a more ballistic transport resulting in tilting of the columns instead of the normally observed growth perpendicular to the substrate, that has to be connected solely to the heteroepitaxial relationships.

\subsection{X-ray diffraction}

The diffraction patterns collected for all the fabricated samples are presented in Fig. 6 .

The pattern for sample 1 confirms the porous character of the sample evidencing a strongly polycrystalline structure as well as hybrid phase composition with both $\mathrm{Zn}(10.1,10.0,10.2$, and 10.3 , or 11.0) and $\mathrm{ZnO}(10.0$, 10.1, and 11.0) presence.

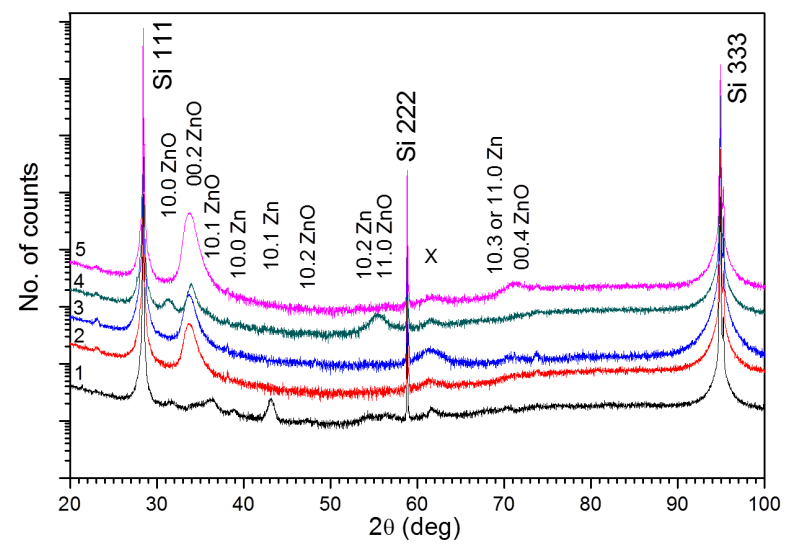

Fig. 6. XRD patterns of the samples produced in the experiment. Respective sample numbers are denoted on the left hand side of each pattern.

Please note that apart from the substrate Si peaks and film $\mathrm{ZnO}$ and $\mathrm{Zn}$ peaks, there is a broad peak at about $61^{\circ}$ denoted by $\mathrm{X}$. This peak could not be identified as related to any known $\mathrm{Zn}$ or $\mathrm{ZnO}$ plane. As the films were deposited without intentional substrate heating, a $\mathrm{Zn}-\mathrm{Si}$ phase could not have formed in volume sufficient for observation in the $\theta-2 \theta$ geometry. Most probably the peak is related to a crystallographic imperfection or impurity in the Si substrate.

In the case of samples 2, 3 and 5 the $\mathrm{ZnO}$ films have a preferred 00.2 orientation. The peaks are however quite broad, their FWHM being around $1.5^{\circ}$, indicating that the relevant lattice planes in the sample are not perfectly parallel to one another and that a slight tilt is present. This condition is often connected to columnar growth, where a group of planes in one column is tilted with respect to the same group of planes in another column.

The highest intensity of the $00.2 \mathrm{ZnO}$ line is observed for sample 5 , however this is a thickness-related effect, as the $\mathrm{ZnO}$ film in sample 5 is roughly twice as thick as the ones in sample 2 or 3 (see Fig. 5). As a thicker film leads to a more pronounced diffraction signal than a thinner one, any comparisons of diffraction line intensities should be performed only between patterns recorded for films of similar thicknesses. Bearing this in mind and seeing that the patterns for samples 2 and 3 are almost identical in the area of the 00.2 line, it can be noted that the change in the oxygen content in the sputtering mixture from $17 \%$ to $60 \%$ did not influence the crystalline structure of the film grown in RF mode, which is in agreement with the determined rather static character of $n_{\mathrm{e}}$ as a function of oxygen concentration changes.

Qualitative comparison of the diffraction patterns for samples 4 and 5 leads to a conclusion that in the case of DC sputtering the same change in oxygen content leads to a significantly different sample structure. The film in sample 4, grown at low oxygen content yielding a plasma density $n_{\mathrm{e}}=1.9 \times 10^{17} \mathrm{~m}^{-3}$ exhibits polycrystalline character with reflections from 10.0, 00.2, ad 11.0 $\mathrm{ZnO}$ planes. 
On the other hand, the film in sample 5 grown at high oxygen content leading to a 2.5-times lower plasma density $\left(n_{\mathrm{e}}=0.8 \times 10^{17} \mathrm{~m}^{-3}\right)$ has a single 00.2 orientation.

It could be therefore concluded that in the case of DC sputtering the plasma density influences the crystalline structure of the films significantly, with high plasma density leading to a growth of polycrystalline $\mathrm{Zn}-\mathrm{ZnO}$ films, medium plasma density leading to a growth of polycrystalline $\mathrm{ZnO}$ films and low plasma density — to the growth of single orientation films. However, it has to be noted here that for the RF mode, the medium density plasma is the only one available in this range of oxygen content and pressure, nevertheless it leads to the growth of single orientation films. Therefore, a simple model correlating the plasma density to the crystal structure of the deposited films does not work.

As seen for the RF case, the model leading to the growth of single orientation films can be supplemented by taking into account the effects of high plasma-substrate bias, i.e. high energy of the impeding ions (see Fig. 4). It is known in the reactive sputter growth of some dense materials, such as TiN, that only under significant substrate bias leading to increased ion bombardment, the films will have the desirable orientation and high density $[6,7]$. This may be also the case in $\mathrm{ZnO} \mathrm{RF}$ deposition. In $\mathrm{DC}$ mode we observe no process conditions leading to the simultaneous creation of a medium-density plasma with high substrate bias. High bias is only present in the case of porous film growth, which was discussed in the previous section. In DC mode the low-density plasma conditions are favourable for the growth of the dense $\mathrm{ZnO}$ films due to the high kinetic energy of the incoming species, however arising not from a high substrate bias, but from less scattering collisions on the way from the target.

\section{Conclusions}

The electrical and optical parameters of $\mathrm{Ar}-\mathrm{O}-\mathrm{Zn}$ discharges in the processes of DC and RF magnetron sputtering from $\mathrm{Zn}$ and $\mathrm{ZnO}$ targets were studied in order to examine the mechanism of porous $\mathrm{Zn}$ film formation. We observed that for high density plasmas $\left(\approx 7 \times 10^{17} / \mathrm{m}^{3}\right)$ the fabricated films are porous and polycrystalline $\mathrm{Zn}$ whereas for lower density plasmas the films are columnar and polycrystalline $\mathrm{ZnO}$ with a dominating orientation of 00.2. It is also worth pointing out that for low density plasmas $\left(\approx 0.8 \times 10^{17} / \mathrm{m}^{3}\right)$, the columnar structure was found to be tilted with relation to the substrate surface, while normally the columns grow perpendicular to it. This effect was connected to the changes in mean free path of the sputtered species with changes in the plasma density. During porous film growth, a significant energy of the impinging ions was registered which is also expected to take a relevant part in the determination of the porous morphology as discussed. It was shown that $\mathrm{DC}$-mode reactive $\mathrm{Zn}$ sputtering allows to cover a broader range of plasma densities, whereas RF-mode re- active $\mathrm{ZnO}$ sputtering showed medium-valued densities regardless of the process conditions.

\section{References}

[1] B. O'Regan, M. Grätzel, Nature 353, 737 (1991).

[2] H. Cheng, W. Chiu, C. Lee, S. Tsai, W. Hsieh, J. Phys. Chem. C 112, 16359 (2008).

[3] X.D. Wang, Y. Ding, C.J. Summers, Z.L. Wang, J. Phys. Chem. B 108, 8773 (2004).

[4] S.-H. Ko, D. Lee, H.-W. Kang, K.-H. Nam, J.-Y. Yeo, S.-J. Hong, C.P. Grigoropoulos, H.-J. Sung, Nano Lett. 11, 666 (2011).

[5] M.A. Borysiewicz, E. Dynowska, V. Kolkovsky, J. Dyczewski, M. Wielgus, E. Kamińska, A. Piotrowska, Phys. Status Solidi A 209, 2463 (2012).

[6] J.-E. Sundgren, B.-O. Johansson, H.T.G. Hentzell, S.-E. Karlsson, Thin Solid Films 105, 385 (1983).

[7] S. Gangopadhyay, R. Acharya, A.K. Chattopadhyay, S. Paul, Vacuum 84, 843 (2010). 